\title{
Relating irritability and suicidal ideation using mood and anxiety
}

\author{
Gin Malhi, ${ }^{1,2}$ Erica Bell, ${ }^{\oplus 1,2}$ Pritha Das, ${ }^{1,2}$ Tim Outhred ${ }^{1,2}$
}

\begin{abstract}
${ }^{1}$ Sydney Medical School, The University of Sydney, Sydney, New South Wales, Australia ${ }^{2}$ Department of Psychological Medicine, The University of Sydney Northern Clinical School, St Leonards, New South Wales, Australia
\end{abstract}

Correspondence to Professor Gin Malhi, University of Sydney Sydney Medical School, Sydney, NSW 2065, Australia; gin.malhi@sydney. edu.au

Received 5 June 2019 Accepted 17 June 2019 Published Online First 27 June 2019

\section{Check for updates}

(C) Author(s) (or their employer(s)) 2019. No commercial re-use. See rights and permissions. Published by BMJ.

To cite: Malhi G, Bell E,

Das $\mathrm{P}$, et al. Evid Based

Mental Health

2019:22:95-99.

\section{ABSTRACT}

Background Suicide is common in the context of depression and bipolar disorders, but there remains a lack of understanding as to how suicide ideation, a common symptom of mood disorders, progresses to suicidal behaviour. Irritability, a feature of some types of depression, is thought to contribute to the development of suicidal behaviour, but these associations are not well established.

Objective To examine the relationship between irritability and suicide ideation according to the subtype of depression expressed in patients with mood disorder. Methods 75 patients with mood disorders seen at the CADE (Clinical Assessment Diagnostic Evaluation) Clinic underwent clinical assessment for suicidal ideation (Paykel Suicide Scale), symptom severity (Young Mania Rating Scale (YMRS), Hamilton Rating Scale for Depression (HAM-D) (anxious depression), MontgomeryÅsberg Depression Rating Scale (MADRS) (melancholic depression)) and irritability (item 5 of the YMRS).

Findings Interestingly, irritability correlated with mania $(r=0.734, p<0.001$ (YMRS)) and depressive symptom scores ( $r=0.369, p<0.001$ (MADRS); $r=0.477, p<0.001$ (HAM-D)), which in turn correlated with suicide ideation scores ( $r=0.364, p<0.01$ (MADRS); $r=0.275, p=0.017$ (HAM-D)). However, despite this indirect association, there was no direct correlation between irritability and suicide ideation ( $r=0.050, p>0.05)$.

Conclusions The nature of the relationship between irritability and suicidal ideation is determined by the emotional context within which irritability operates. Clinical implications Findings suggest that rather than examining irritability alone, consideration of the subtype of depression, especially that of anxious depression, should be paramount in assessing suicide risk.

\section{BACKGROUND}

Suicidal ideation is a common feature of mood disorders. It needs to be addressed urgently in both clinical settings and in the general population because rates of suicide, in developed countries such as Australia, have remained virtually unchanged for the past 15 years despite extensive research and the best efforts of preventative programmes. ${ }^{1}$ Even more worrisome, the rates of suicide within clinical populations, such as those with mood disorders, are significantly higher than the general population (20-30 times the risk of the general population in bipolar disorder). ${ }^{2}$ Hence, considerable resources have been dedicated to determining the key suicide-related factors that may hold predictive

\section{Summary box}

What is already known about this subject?

- Suicidal ideation is a common feature of mood disorders, particularly depression.

- Depression is a heterogeneous condition, with anxious depression and melancholic depression being two key subtypes.

- Irritability is a common symptom of mood disorders and is linked to suicidal ideation.

- As yet no research has specifically examined the way in which irritability operates within anxious and melancholic subtypes of depression to contribute to suicidal ideation.

What are the new findings?

- Within the two depression scales used, irritability correlated with items of suicidal ideation in one scale but not another.

- Irritability showed no direct correlation with scores on a measure of suicidal ideation.

- Irritability demonstrated an indirect correlation with suicidal ideation.

- It appears that the relationship between irritability and suicidal ideation is dependent on the mood context within which it operates.

How might it impact on clinical practice in the foreseeable future?

- These findings suggest that in clinical practice, consideration of the subtype of depression, rather than irritability alone, should be paramount in assessing suicide risk.

value in identifying at-risk individuals. ${ }^{3}$ But thus far, little discernible impact has been made.

Given the prevalence of suicidal ideation in mood disorders, a useful strategy in understanding the role of suicide-related factors is to examine these thoughts in the context of these illnesses. This approach also takes advantage of the fact that depression is a heterogeneous condition and that the relationship of its various subtypes with suicidal ideation varies accordingly, and therefore by examining suicidal ideation in the context of depression the factors associated with suicidal thinking can be partitioned. ${ }^{4-6}$ For example, among the subtypes of depression, anxious depression appears to confer significantly higher risk for suicide than depression in which anxious features are absent. ${ }^{4}$ Of note, within this subtype of depression, individual syndromes consisting of psychomotor agitation, restlessness and irritability feature prominently. ${ }^{5}$ 
Another subtype of depression that features psychomotor changes is melancholia, which is characterised by low energy, anhedonia and loss of interest. ${ }^{6}$ Clinically, melancholia contrasts with anxious depression, but suicidal ideation is again a prominent feature. However, importantly, the motivating factors driving suicidal ideation and behaviour differ according to depressive subtype. ${ }^{8}$

Interestingly, even though major depression is clinically indistinguishable from depression in bipolar disorder, suicide, which occurs predominantly in the depressive phase, is more common in bipolar disorder. Hence mapping the development of suicidal thinking in bipolar disorders-as has been done recently-is informative and this has led to the identification of some key stages in the process (see Malhi et $a l^{3}$ ). In the context of bipolar disorder, the symptom of suicidal ideation, if unchecked, is liable to progress to suicidal behaviours, attempts, and in some cases completed suicide. This progression is complex and multifaceted, and is currently not completely understood. However, research has noted the differential impact of distal and proximal factors, which affect the severity of suicidal thoughts and behaviours, with each factor producing variable impact at different points along the pathway to suicide.

One such important factor that has gained interest in recent years is the symptom of irritability, which has been posited to increase the likelihood of transitioning from suicidal ideation to suicide attempt in the context of psychiatric disorders. ${ }^{9}$ Although irritability is primarily considered a symptom of mania and is included in scales measuring manic symptoms, it is during depressive episodes of mood disorders that suicidal ideation and behaviours are more likely to occur. Furthermore, irritability is common to both anxious and melancholic depression, and is also correlated to suicidal ideation within these clinical populations. ${ }^{10}$ However, despite irritability being present within both subtypes of depression (linking depression to suicide), the specific manner in which irritability functions within these two distinct presentations is not known. We postulated that differences in how depression moderates the link between irritability and suicide may differ according to depressive subtype, and that the nature of these differences may provide valuable insights into suicidal ideation.

\section{OBJECTIVE}

This study aimed to better understand the relationship between irritability and suicide by exploiting its differential moderation by different depressive subtypes. To achieve a more granular analysis than that conducted to date, the study comprehensively assessed both the subtype of depression (using two measures) and suicidal ideation (using a dedicated scale).

\section{METHODS}

Seventy-five patients aged 18-65 years with a diagnosis of a mood disorder (either bipolar disorder or major depressive disorder) participated in the study from 2016 to 2017. Subjects were recruited to the study as they presented sequentially to the CADE (Clinical Assessment Diagnostic Evaluation) Clinic, with those that met a diagnosis of a mood disorder included and those without a mood disorder excluded. All subjects underwent a detailed and structured clinical assessment conducted by a psychiatrist to determine they met the criteria for a DSM-5 (Diagnostic and Statistical Manual for Mental Disorders - 5th Revision) diagnosis for a mood disorder and to assess suicidal activity, depressive symptom severity and manic symptom severity. Subjects included in the study had not experienced medication changes, or received electroconvulsive therapy or psychotherapy within the preceding month.

Depression was rated using two scales: the 21-item Hamilton Rating Scale for Depression (HAM-D), ${ }^{11}$ which categorises the severity of depression scores as mild (8-13), moderate (14-18), severe (19-22) and very severe (23+), and the Montgomery-Åsberg Depression Rating Scale (MADRS), ${ }^{12}$ which rates the severity of depression in patients with mood disorders using 10 items, and categorises depression as mild (7-19), moderate (20-34) and severe $(34+)$. Of note, although the MADRS typically correlates highly with scores on the HAM-D, the former is typically more attuned to melancholic features of depressionreflecting a more melancholic subtype and showing greater sensitivity to medications ${ }^{13}$ than the HAM-D, which is generally more sensitive to anxiety. ${ }^{5}$ The use of two depression scales allowed for comprehensive assessment of the various features of depression within the study cohort that contained a variety of mood disorder presentations. Furthermore, using both the HAM-D and the MADRS maximised the possibility of capturing both anxious and melancholic depressive features in the present cohort.

Manic symptoms were rated using the Young Mania Rating Scale (YMRS), ${ }^{14}$ which comprises 11 items scored either from 0 to 4 or from 0 to 8 . Thus four items (irritability, speech, thought content and disruptive/aggressive behaviour) are given twice the weight of other items to compensate for poor cooperation. The total score extends from 0 to 60 , with higher scores indicating greater severity. Item 5 of the YMRS was used as a measure of irritability in each subject during a clinical interview conducted by a psychiatrist as this study examined a clinical population, whose ratings of irritability required being assessed in the context of a mood disorder.

To evaluate suicidal ideation, subjects were assessed using the Paykel Suicide Scale, ${ }^{15}$ which is a five-item questionnaire. This questionnaire assesses thoughts of death, suicidal ideation and suicide attempts and is subjectively reported by participants using a 5-point scale ranging from 'never' to 'always'. Thus, total scores can range from 5 to 25 , with higher scores indicating greater intensity of suicidal ideation. The Paykel was used in addition to the HAM-D and the MADRS because, although both the depression rating scales contain items which measure suicidal ideation, these particular items focus on different aspects of suicidal ideation (eg, the HAM-D considers suicide attempts, while the MADRS does not) and therefore they do not provide a comprehensive assessment of the construct. Hence, the Paykel was used to provide a more complete assessment of suicidal ideation, which would then allow interrogation of its relationship with other factors being examined.

\section{Statistical analysis}

Statistical analysis was conducted using IBM SPSS Statistics (V.24) for Windows. Scores on the irritability item of the YMRS scale were compared with the overall scores on each of the YMRS, HAM-D, MADRS and Paykel scales as well as individual items. Pearson's correlation analyses were conducted to produce a correlation matrix to assess whether a significant $(p<0.05)$ relationship did exist between the YMRS irritability score and any item assessing suicidal ideation. This allowed for assessment of the relationship between overall scores on the Paykel questionnaire and the total YMRS, HAM-D and MADRS scores. 
Table 1 Descriptive statistics of mood measures and suicidality items

\begin{tabular}{llcrl}
\hline & Minimum & Maximum & Mean & \multicolumn{1}{c}{ SD } \\
\hline Irritability & 0.00 & 4 & 2.00 & 1.19 \\
YMRS total & 0.00 & 11.00 & 3.80 & 2.51 \\
MADRS total & 0.00 & 40.00 & 22.67 & 8.52 \\
$\begin{array}{l}\text { MADRS item 10 (suicidal } \\
\text { thoughts) }\end{array}$ & 0.00 & 6.00 & 1.75 & 1.12 \\
$\begin{array}{l}\text { HAM-D total } \\
\text { HAM-D item 3 }\end{array}$ & 0.00 & 30.00 & 16.89 & 6.211 \\
$\begin{array}{l}\text { (suicidality) } \\
\text { Paykel total }\end{array}$ & 0.00 & 3.00 & 0.88 & 0.753 \\
\hline
\end{tabular}

HAM-D, Hamilton Rating Scale for Depression; MADRS, Montgomery-Åsberg

Depression Rating Scale; YMRS, Young Mania Rating Scale.

\section{FINDINGS}

\section{Irritability}

Scores on the YMRS irritability item ranged from 0 'Absent' $(n=13)$ to 4 'Irritable at times during interview; recent episodes of anger or annoyance on ward' $(n=13)$ with a mean score of 2 'subjectively increased' $(n=49)$.

Details of the maximum, minimum, mean and SD of scores of irritability, mania, overall depression, overall suicidal ideation and individual suicidality items of depression scales are given in table 1 .

\section{Correlations between irritability and total scores}

Overall, the irritability score demonstrated a significant correlation with the total HAM-D score $(\mathrm{r}=0.477, \mathrm{p}<0.001)$. Additionally, the irritability score correlated significantly with the individual items of the HAM-D, including item 3 which assessed suicidal ideation $(\mathrm{r}=0.242, \mathrm{p}=0.036)$.

Overall, scores for the irritability item of the YMRS correlated significantly with both the total MADRS scores $(r=0.369$, $\mathrm{p}=0.001$ ) and with each individual item on the MADRS, with the exception of item 10 which assesses suicidal ideation $(\mathrm{r}=0.184, \mathrm{p}=0.114)$ (table 2).

Overall, the analysis failed to detect a significant relationship between irritability and total Paykel score. The analysis also failed to detect a significant relationship between the irritability score and the score of any individual Paykel scale item.

\section{Correlations between suicidal ideation and overall scores}

Overall, the total scores on the Paykel questionnaire correlated significantly with the total scores on both the MADRS $(r=0.364$, $\mathrm{p}<0.01)$ and the HAM-D $(\mathrm{r}=0.275, \mathrm{p}=0.017)$. However, the analysis did not find a significant relationship between the overall YMRS score and the total Paykel score.

\section{DISCUSSION}

Using carefully collected data, we aimed to examine the seemingly nebulous relationship between irritability and suicidal ideation. Irritability and suicidal ideation appeared to be differentially linked depending on the measure used.

The suicidal ideation item of the HAM-D correlated with irritability; however, its equivalent in the MADRS did not. Additionally, no relationship was detected between irritability scores and total suicide scores on the Paykel questionnaire-itself a targeted comprehensive assessment of suicidal ideation. As seen in figure 1 , the differential relationship between irritability and suicidal ideation seen in our data set may be explained by the 'qualities' (or nature) of the 'emotional context' in which it appears, in other words the mood milieu.

The two depression measures used in the present study identified the heterogeneity of the influence of mood on irritability. Clinically, the HAM-D detects anxious depression and its various features, and as such it has been used to investigate agitated and anxious depression. ${ }^{16}$ Conversely, the MADRS is sensitive to melancholic features of depression. The differential relationship seen between irritability and suicidal ideation scores across the MADRS and the HAM-D suggests that, although irritability is related to depression, which in turn is related to suicide, the association between irritability and suicide is very much determined by the particular subtype of depression via which its influence is being mediated. Furthermore, this influence is subject to additional moderation by the presence of anxiety, as captured by the HAM-D.

Given that suicidal ideation is a common feature of mood disorders, but does not always eventuate in suicidal behaviour/ attempts, there must be other factors that influence progression towards suicide. Research in youth has suggested that irritability plays a role in this progression, because its presence increases the likelihood of suicidal ideation leading to an attempt-indicating perhaps that it is acting as a catalyst for this process as opposed to being a trigger for its inception. ${ }^{9} 1017$ Specifically, this means that irritability requires mood to provide it with the necessary emotional context, which then perhaps generates an 'irritable mood' and it is this that has a transformative suicidogenic effect and is therefore linked to suicidal ideation.

The associations found in our study suggest that in the context of anxious depression irritability is more conducive to suicidal ideation. Conversely, while melancholia is associated with suicide, it does not facilitate the contribution of irritability to suicidal ideation to the same extent as anxious depression. In other words, while depression as a whole is a well-established risk factor for suicide, it is when depressive features are coupled with features of anxiety and irritability

Table 2 Pearson's correlation matrix between irritability, mania, depression and suicidal ideation symptom scores

\begin{tabular}{llllllll}
\hline & 1 & 2 & 3 & 4 & 5 & 6 \\
\hline 1. Irritability & 1 & & & & & & \\
2. YMRS total & $0.734^{* *}$ & 1 & & & & & \\
3. HAM-D total & $0.477^{* *}$ & $0.469^{* *}$ & 1 & $0.448^{* *}$ & 1 & & 1 \\
4. HAM-D suicide & $0.242^{*}$ & 0.151 & $0.800^{* *}$ & $0.493^{* *}$ & 1 & $0.663^{* *}$ & 1 \\
5. MADRS total & $0.369^{* *}$ & $0.332^{* *}$ & $0.592^{* *}$ & 1 \\
6. MADRS suicide & 0.184 & 0.107 & $0.548^{* *}$ & $0.768^{* *}$ & $0.364^{* *}$ & \\
7. Paykel & 0.050 & 0.006 & $0.275^{*}$ & $0.723^{* *}$ & & & \\
\hline
\end{tabular}

${ }^{*}$ Correlation is significant at the 0.05 level (two-tailed).

${ }^{*}$ Correlation is significant at the 0.01 level (two-tailed).

HAM-D, Hamilton Rating Scale for Depression; MADRS, Montgomery-Åsberg Depression Rating Scale; YMRS, Young Mania Rating Scale. 


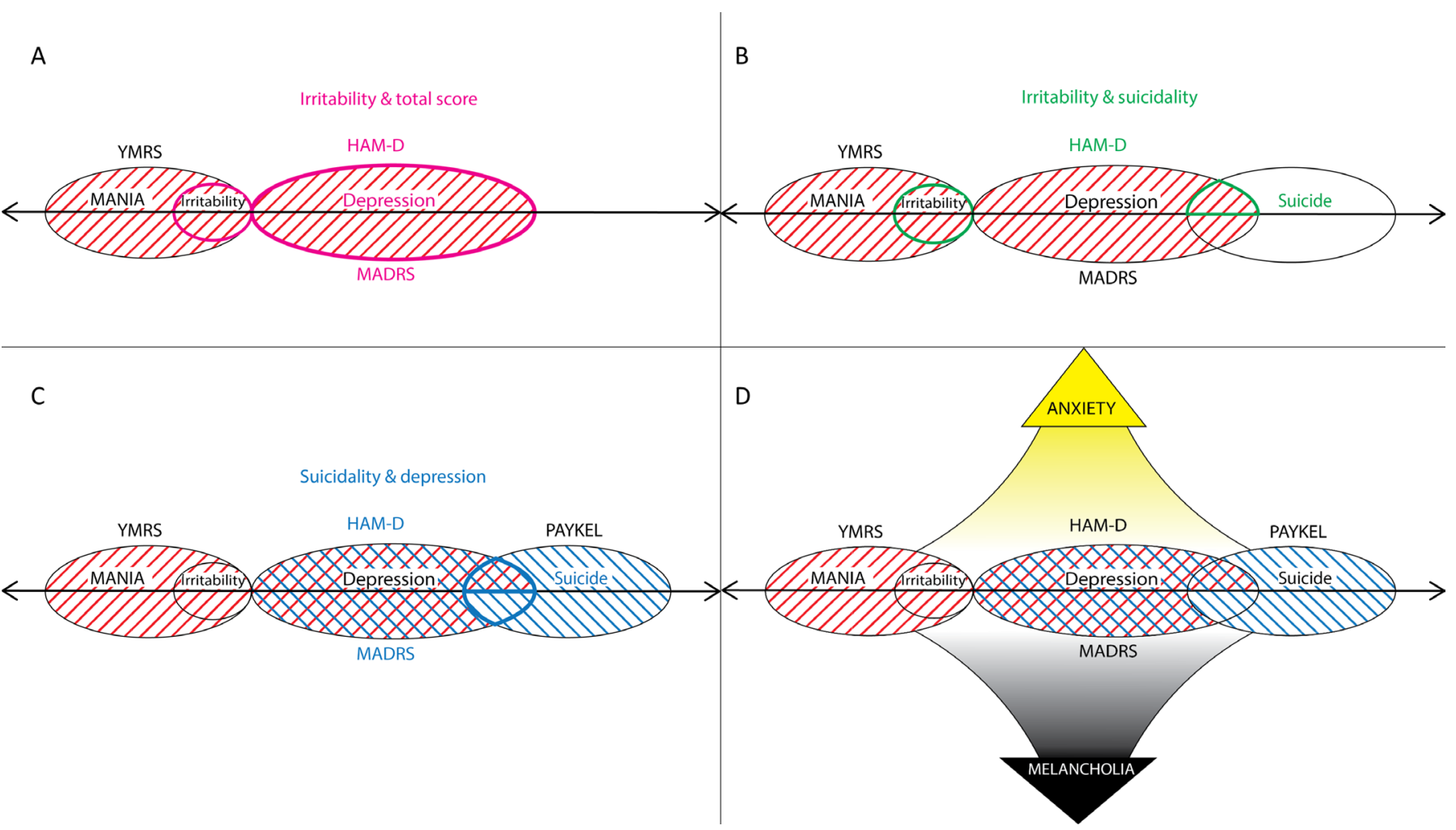

Figure 1 Schematic diagram of correlations between measures and the domains in which the psychometric assessments are most sensitive. In quadrant $A$, correlations (pink) are demonstrated between the irritability item of the YMRS (red stripes) and the YMRS, HAM-D and MADRS total scores. Quadrant B demonstrates correlations (green) between irritability scores (YMRS item) and suicidal ideation items within mood scales. There is a correlation with the HAM-D suicide ideation item, but not with the suicide ideation item within the MADRS. Quadrant C demonstrates correlations (blue) between Paykel suicidality scores (blue stripes) and total HAM-D and MADRS scores as well as ratings on the suicidality items within both depression scales. Quadrant D demonstrates the domains of anxiety and melancholia within which the HAM-D and MADRS are most sensitive. HAM-D, Hamilton Rating Scale for Depression; MADRS, Montgomery-Åsberg Depression Rating Scale; YMRS, Young Mania Rating Scale.

that the relationship to suicide is especially enhanced. Furthermore, irritability alone may not confer additional suicide risk, and that it is only once irritability occurs in the context of depression and anxiety that this risk is realised.

\section{Limitations}

A potential limitation of this study is the relatively low scores of irritability on item 5 of the YMRS, as seen in table 1. Although one could argue that this would reduce the effects seen of irritability on measures of suicide, it is worth noting the large increases in severity between scores of irritability on the YMRS. For example, a score of 2 would suggest that irritability has 'subjectively increased', whereas a score of 4 is indicative of being 'irritable at times during interview; recent episodes of anger or annoyance on ward'. ${ }^{14}$ This escalation from subjective awareness of one's own irritability to being externally angry on the ward or during a short interview is a substantial increase in irritability to be captured in one increment. Therefore, the sensitivity of future examinations of irritability could be greatly improved by the development of new assessments for this construct, as recent investigations have demonstrated that measures used currently are inadequate. ${ }^{18}$

This study is clearly preliminary and correlational in nature and does not use a comparison group; therefore, a causal relationship cannot be inferred from the present results alone. But what the current findings do provide is an avenue for further investigation into the intricate relationship between irritability and suicidal ideation and how this relationship, which likely evolves over time, differs depending on the context of mood in which it operates. This modest but novel study thus opens the possibility of examining the construct of suicidal ideation anew, with greater consideration being given to the contributory role of irritability and its interaction with anxiety in the context of mood.

\section{CLINICAL IMPLICATIONS}

The findings from the present study suggest that rather than examining irritability alone, consideration of the subtype of depression, particularly those suffused with anxiety, should be paramount in assessing suicide risk. This is in keeping with clinical findings where individuals whose depression is characterised by anxiety and irritability are at higher risk of suicide than those with melancholic features. In light of these findings, it is important that future studies examine irritability as a risk factor for suicidal ideation, and consider the context of mood and anxiety in which it operates.

\section{Future research}

Future research should be premised on the present results and examine irritability specifically within the context of its interactions with mood, as this appears to be instrumental in shaping its clinical impact. By using mood as a frame of reference on which the effect of irritability can be adhered, its influence on suicidal ideation can be comprehensively appreciated. 
Contributors GM, PD and TO developed, planned and conducted the study. EB conducted the analysis of the data. GM, EB, PD and TO contributed to the writing of the finalised article.

Funding This research is supported by grants PRG-0-090-14 and SRG- 0-089-16 awarded to GM, PD and TO by the American Foundation for Suicide Prevention, as well as the Australian Rotary Health, the Sydney Medical School Foundation, SPARK Sydney, the Ramsay Health Research and Teaching Fund, and an NHMRC Program Grant (APP1073041).

Disclaimer The content is solely the responsibility of the authors and does not necessarily represent the official views of the American Foundation for Suicide Prevention.

Competing interests GM has received grant or research support from NHMRC, NSW Health, The University of Sydney, American Foundation for Suicide Prevention, Australian Rotary Health, AstraZeneca, Eli Lilly \& Co, Organon, Pfizer, Servier and Wyeth; has been a speaker for AstraZeneca, Eli Lilly \& Co, Janssen Cilag, Lundbeck, Pfizer, Ranbaxy, Servier and Wyeth; and has been a consultant for AstraZeneca, Eli Lilly \& Co, Janssen Cilag, Lundbeck and Servier. EB, PD and TO have no conflicts of interest to report.

Patient consent for publication Not required.

Ethics approval The University of Sydney and Royal North Shore Hospital.

Provenance and peer review Not commissioned; externally peer reviewed.

\section{REFERENCES}

1. Jorm AF. Australia's 'Better Access' scheme: Has it had an impact on population mental health? Aust N Z J Psychiatry 2018;52:1057-62.

2. Pompili M, Gonda X, Serafini $G$, et al. Epidemiology of suicide in bipolar disorders: a systematic review of the literature. Bipolar Disord 2013;15:457-90.

3. Malhi GS, Outhred T, Das P, et al. Modeling suicide in bipolar disorders. Bipolar Disord 2018;20:334-48.

4. Goldberg D, Fawcett J. The importance of anxiety in both major depression and bipolar disorder. Depress Anxiety 2012;29:471-8.
5. Fava M, Alpert JE, Carmin CN, et al. Clinical correlates and symptom patterns of anxious depression among patients with major depressive disorder in STAR ${ }^{*} D$. Psychol Med 2004;34:1299-308.

6. Parker G, Tavella G, Hadzi-Pavlovic D. Identifying and differentiating melancholic depression in a non-clinical sample. J Affect Disord 2019;243:194-200.

7. Fletcher K, Parker G, Paterson A, et al. Anhedonia in melancholic and non-melancholic depressive disorders. J Affect Disord 2015;184:81-8.

8. Rihmer Z, Kiss K. Bipolar disorders and suicidal behaviour. Bipolar Disord 2002:4:21-5.

9. Benarous $X$, Consoli A, Cohen D, et al. Suicidal behaviors and irritability in children and adolescents: a systematic review of the nature and mechanisms of the association. Eur Child Adolesc Psychiatry 2019;28:667-83.

10. Orri M, Perret LC, Turecki G, et al. Association between irritability and suicide-related outcomes across the life-course. Systematic review of both community and clinical studies. J Affect Disord 2018:239:220-33.

11. Hamilton M. A rating scale for depression. J Neurol Neurosurg Psychiatry 1960:23:56-62.

12. Montgomery SA, Asberg M. A new depression scale designed to be sensitive to change. Br J Psychiatry 1979;134:382-9.

13. Santen G, Danhof M, Della Pasqua O. Sensitivity of the Montgomery Asberg Depression Rating Scale to response and its consequences for the assessment of efficacy. J Psychiatr Res 2009;43:1049-56.

14. Young RC, Biggs JT, Ziegler VE, et al. A rating scale for mania: reliability, validity and sensitivity. Br J Psychiatry 1978;133:429-35.

15. Paykel ES, Myers JK, Lindenthal JJ, et al. Suicidal feelings in the general population: a prevalence study. Br J Psychiatry 1974;124:460-9.

16. Tondo L, Vázquez GH, Pinna $\mathrm{M}$, et al. Characteristics of depressive and bipolar disorder patients with mixed features. Acta Psychiatr Scand 2018;138:243-52.

17. Orri M, Galera C, Turecki G, et al. Pathways of association between childhood irritability and adolescent suicidality. J Am Acad Child Adolesc Psychiatry 2019;58:99-107.

18. Toohey MJ, DiGiuseppe R. Defining and measuring irritability: construct clarification and differentiation. Clin Psychol Rev 2017:53:93-108. 